\title{
S3. $z$-score
}

As a further step to test the robustness of our results, we standardized the cAIx by calculating a $z$-score. The $z$-score was calculated as cAIx minus the mean of the population divided by the standard deviation, and it took account of heterogeneities between patients such as age and gender. A $z$-score of zero would have implied that cAIx did not differ from the population mean. Figure 2 (below) presents the results as a box plot: there was a negative $z$-score for patients with AAA, and a slightly negative $z$-score for patients with both PAD and AAA. These results indicated that the cAIx was lower for patients with aneurysm than for patients with PAD.

\section{Figure 2}

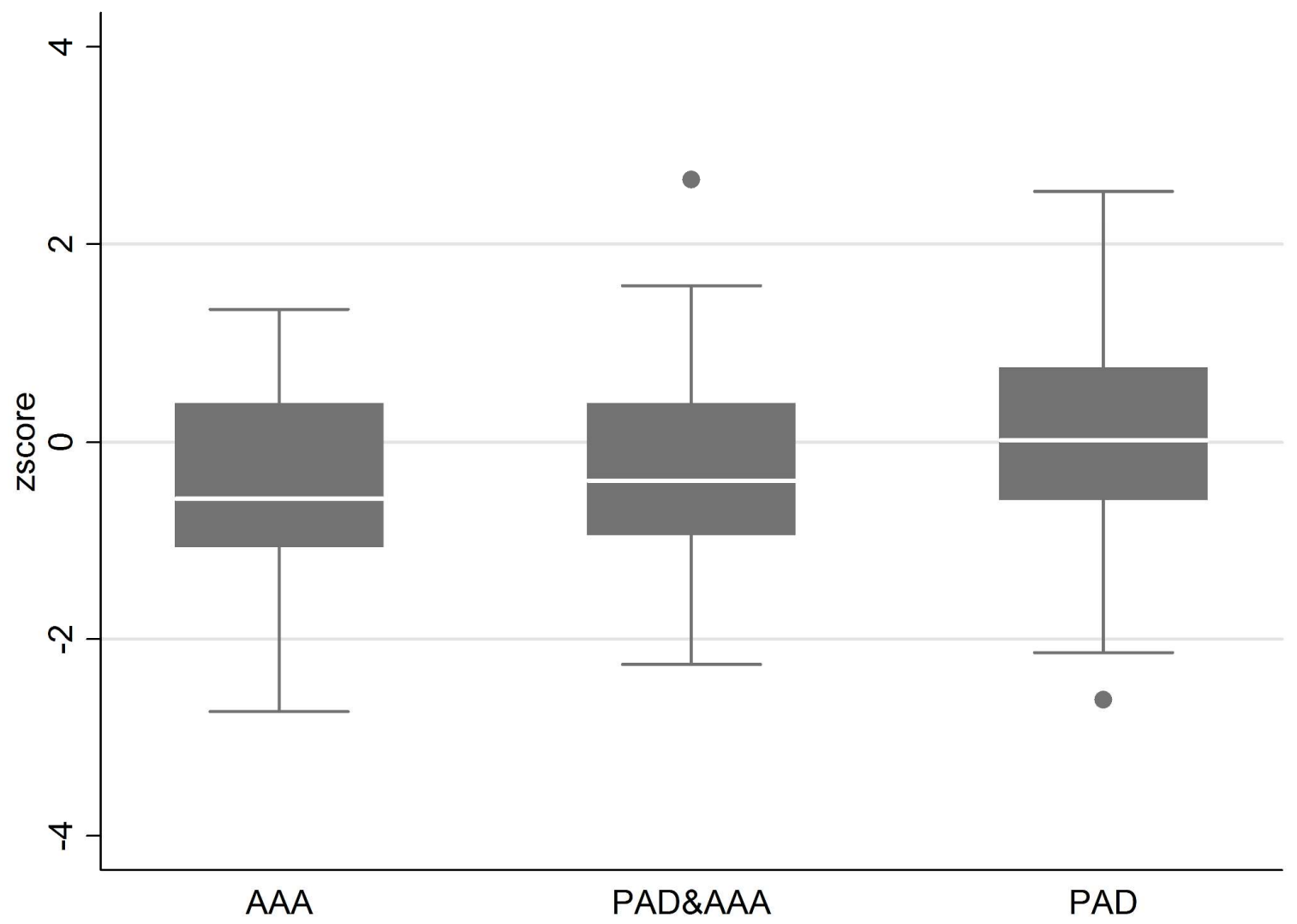

